\title{
Self-trapping of optical beams in photorefractive media
}

\author{
Bruno Crosignani \\ Dipartimento di Fisica, Università dell'Aquila, L'Aquila, Italy, and Fondazione Ugo Bordoni, Rome, Italy \\ Mordechai Segev and Doruk Engin \\ California Institute of Technology, Pasadena, California 91125 \\ Paolo Di Porto \\ Dipartimento di Fisica, Università dell'Aquila, L'Aquila, Italy, and Fondazione Ugo Bordoni, Rome, Italy
}

Amnon Yariv

California Institute of Technology, Pasadena, California 91125

Greg Salamo

Department of Physics, University of Arkansas, Fayetteville, Arkansas 72701

Received July 16, 1992; revised manuscript received October 9, 1992

\begin{abstract}
We study the possibility of self-trapping of an optical beam in a photorefractive medium under the combined influence of diffraction and self-scattering (two-wave mixing) of its spatial frequency components. We investigate the spectrum of solutions for the resulting photorefractive spatial solitons and discuss their unique properties. Design considerations and material requirements for experimental realization of these solitons, together with specific examples, are given.
\end{abstract}

\section{INTRODUCTION}

Self-trapping of light beams in nonlinear self-focusing Kerr media has been explored intensively during the past three decades. The self-trapped beams, often in the form of spatial solitons, evolve from nonlinear changes in the refractive index of the material that are induced by the intensity distribution of the light when the confining effect of the refractive index exactly compensates for the defocusing effect of diffraction. After self-focusing of light beams ${ }^{1}$ in the presence of the optical Kerr effect was observed experimentally, a theoretical solution for the resulting spatial solitons was found. ${ }^{2,3}$ This solution was followed by demonstrations of self-trapping in vapors ${ }^{4}$ and liquids, ${ }^{5}$ and finally, within the past few years, spatial solitons in solid (glass) media were also observed. ${ }^{6}$ However, the index changes needed for Kerr-like spatial solitons require high intensities, intensities often exceeding $1 \mathrm{MW} / \mathrm{cm}^{2}$.

In a recent paper ${ }^{7}$ we suggested a new type of spatial soliton that is associated with the photorefractive (PR) effect in a crystal. The intensity profile of the beam modulates the refractive index by means of the PR effect, which results in an exact compensation for the effects of diffraction and causes the light beam to propagate with an unvarying profile. These new solitons arise from the nonlocal PR effect rather than from the local Kerr effect. They can be generated even at moderate light intensities, since the efficiency of the PR effect is independent of the absolute light intensity.
In this paper we provide a detailed derivation of the equation governing propagation in a PR material and explore the spectrum of its solutions that pertain to distortionless propagation. We discuss the properties of the PR solitons and specify the material requirements and the design considerations for an experimental realization. Finally, we evaluate the minimal nonlinearity that is required for trapping a light beam and consider a specific example of self-trapping in strontium barium niobate (SBN).

\section{BEAM PROPAGATION IN PHOTOREFRACTIVE MEDIA}

The PR solitons are found among the steady-state solutions of the nonlinear wave equation that describe beam propagation in PR media and account for both diffraction and the mutual interaction between each pair of spatial components of the beam. Since the key to this nonlinear scattering process is grating formation by a continuum of Fourier (plane-wave) components of the beam, we cannot resort to the two-plane-wave analysis that is commonly applied to PR materials. Our general formalism accounts for the transverse spatial structure of the beam.

We start by deriving the nonlinear wave equation that describes the propagation of a monochromatic optical beam of a given frequency $(\omega)$ and polarization that is traveling in the positive direction of an arbitrary axis $z$. We assume the absence of nonlinear interactions between orthogonal polarizations (anisotropic scattering ${ }^{8}$ ), so that 
our problem can be reduced to a scalar formulation. Light propagation in nonlinear media can be conveniently described by using coupled-mode theory ${ }^{9}$ applied to the case of unbounded media for which an appropriate set of spatial modes is the continuum of plane waves. ${ }^{10}$ The electric field associated with the light beam that propagates primarily along the $z$ direction is written as

$$
\begin{aligned}
E(\mathbf{r}, z, t)= & \frac{1}{2}\{\exp [i(k z-\omega t)] \\
& \left.\times \int E(\mathbf{q}, \mathbf{r}) \exp \left[i\left(\beta_{q}-k\right) z\right] f(\mathbf{q}, z) \mathrm{d} \mathbf{q}+\text { c.c. }\right] \\
\equiv & \frac{1}{2}[A(\mathbf{r}, z) \exp [i(k z-\omega t)]+\text { c.c. }\},
\end{aligned}
$$

where in the paraxial approximation $\left(k-\beta_{q}\right) \ll k$ and

$$
E(\mathbf{q}, \mathbf{r})=\frac{1}{2 \pi}\left(\frac{\mu_{0}}{\epsilon_{0} n_{1}}\right)^{1 / 2} \exp (i \mathbf{q} \cdot \mathbf{r}) .
$$

$\mathbf{r} \equiv(x, y), k=\omega n_{1} / c$ is the light-wave number, $n_{1}$ is the unperturbed index of refraction in the medium, and $f(\mathbf{q}, z)$ is the spatial frequency (angular) distribution of the complex amplitude $A(\mathbf{r}, z)$. A spatial mode (plane-wave component) is characterized by the projections of its wave vector, $\mathbf{q}$ and $\beta_{q}$, in the transverse (r) and longitudinal $(z)$ directions, respectively, with $\beta_{q}=\left(k^{2}-q^{2}\right)^{1 / 2}$ (where $q$ is restricted to $0 \leq|q| \leq k$ ). Assuming negligible absorption and under rather general conditions, ${ }^{10}$ it is easy to show that, in the presence of a refractive-index distribution $n(\mathbf{r}, z)=n_{1}+\delta n(\mathbf{r}, z), A(\mathbf{r}, z)$ obeys the differential equation

$$
\left(\frac{\partial}{\partial z}-\frac{i}{2 k} \nabla_{\mathbf{r}}^{2}\right) A(\mathbf{r}, z)=\frac{i k}{n_{1}} \delta n(\mathbf{r}, z) A(\mathbf{r}, z) .
$$

The nonlinear term $\delta n(\mathbf{r}, z)$ is obtained by considering the mixing process between two plane waves of the same frequency $\omega$. When only one pair of such plane waves (spatial modes) $\mathbf{q}_{1}$ and $\mathbf{q}_{2}$, of field amplitudes $a_{1}(z)$ and $a_{2}(z)$, is present in the medium, it induces an index grating $\delta n(\mathbf{r}, z)$, which is proportional to the time-averaged interference pattern between the waves. The proportionality coefficient is a complex factor $\hat{\delta} n\left(\mathbf{q}_{1}, \mathbf{q}_{2}\right)$, which represents the PR coupling coefficient between the two plane waves, given the material properties (the orientation of the $\mathrm{PR}$ crystalline medium, its trap density $P_{d}$, its refractive index $n_{1}$, and the dc electric constant $\epsilon_{r}$ ), the externally applied electric field $E_{0}$, and the polarization of the waves. In this simple case, $\delta n(\mathbf{r}, z)$ is ${ }^{11}$

$$
\begin{aligned}
\delta n(\mathbf{r}, z)= & \left(1 / I_{0}\right)\left\{a_{1}(z) \exp \left[i\left(\mathbf{q}_{1} \cdot \mathbf{r}+\beta_{q_{1}} z\right)\right] a_{2}{ }^{*}(z)\right. \\
& \left.\times \exp \left[-i\left(\mathbf{q}_{2} \cdot \mathbf{r}+\boldsymbol{\beta}_{q_{2}} z\right)\right] \hat{\delta}\left(\mathbf{q}_{1}, \mathbf{q}_{2}\right)+\text { c.c. }\right\},
\end{aligned}
$$

where $I_{0}=\left|a_{1}\right|^{2}+\left|a_{2}\right|^{2}$ is the absolute light intensity $\left(I_{0} \gg\left|a_{1} a_{2} *\right|\right)$. Since the perturbation in the refractive index, $\delta n(\mathbf{r}, z)$, is real (no absorption), we get $\delta n\left(\mathbf{q}_{1}, \mathbf{q}_{2}\right)=$ $\widehat{\delta n^{*}}\left(\mathbf{q}_{2}, \mathbf{q}_{1}\right)$. This light-induced index change may be viewed as the sum of two grating components, the real and the imaginary parts of $\hat{\delta} n\left(\mathbf{q}_{1}, \mathbf{q}_{2}\right)$, which correspond to index gratings that are in phase and shifted by $\pi / 2$, respectively, with respect to the light interference grating $a_{1} a_{2} * / I_{0}$. The $\pi / 2$-shifted gratings are responsible for (light) power transfer between the two waves, a phenomenon that can manifest itself as optical amplification, photorefractive oscillation, ${ }^{12}$ and stimulated noise scattering (fanning; see Ref. 13). The in-phase gratings are responsible for nonlinear phase coupling. The real or the imaginary parts of $\delta n\left(\mathbf{q}_{1}, \mathbf{q}_{2}\right)$ are even or odd (respectively) under the exchange $\left(\mathbf{q}_{1}, \mathbf{q}_{2}\right) \rightarrow\left(\mathbf{q}_{2}, \mathbf{q}_{1}\right)$. The index grating $\hat{\delta} n\left(\mathbf{q}_{1}, \mathbf{q}_{2}\right)$ multiplied by $i$ is often called $\gamma$ and yields an intensity-coupling coefficient $\Gamma=2$ $\operatorname{Re}(\gamma)=-2 \operatorname{Im}\left[\hat{\delta n}\left(\mathbf{q}_{1}, \mathbf{q}_{2}\right)\right]$. The index grating $\hat{\delta n}\left(\mathbf{q}_{1}, \mathbf{q}_{2}\right)$ is given by ${ }^{11}$

$$
\hat{\delta n}\left(\mathbf{q}_{1}, \mathbf{q}_{2}\right)=\frac{-\omega}{2 c} n_{1}{ }^{3} r_{\mathrm{eff}}\left(\mathbf{q}_{1}, \mathbf{q}_{2}\right) E_{m}\left(\mathbf{q}_{1}, \mathbf{q}_{2}\right)\left(\mathbf{e}_{1} \cdot \mathbf{e}_{2}{ }^{*}\right),
$$

where $\mathbf{e}_{1}, \mathbf{e}_{2}$ are the polarization vectors of the two interfering plane waves, $r_{\text {eff }}\left(\mathbf{q}_{1}, \mathbf{q}_{2}\right)$ is an effective electro-optic coefficient (which depends on the orientation of the PR crystalline medium), and $E_{m}\left(\mathbf{q}_{1}, \mathbf{q}_{2}\right)$ is the coefficient of the induced space-charge field:

$$
E_{m}\left(\mathbf{q}_{1}, \mathbf{q}_{2}\right)=E_{p}\left(\mathbf{q}_{1}, \mathbf{q}_{2}\right) \frac{E_{d}\left(\mathbf{q}_{1}, \mathbf{q}_{2}\right)-i E_{0}}{E_{0}+i\left[E_{d}\left(\mathbf{q}_{1}, \mathbf{q}_{2}\right)+E_{p}\left(\mathbf{q}_{1}, \mathbf{q}_{2}\right)\right]},
$$

where $E_{0}$ is the externally applied electric field; $E_{p}=$ $e P_{d} /\left(\epsilon_{0} \epsilon_{r} K_{g}\right)$ is the limiting space-charge field and $E_{d}=$ $k_{B} T K_{g} / e$ is the diffusion field ( $e$ is the magnitude of the electron charge, $P_{d}$ is the trap density, $\epsilon_{r}$ is the dielectric constant, $T$ is the temperature, and $k_{B}$ is Boltzmann's constant). Both $E_{d}$ and $E_{p}$ depend on the magnitude and the direction of the interference grating wave vector $\mathbf{K}_{g}=$ $\mathbf{k}_{1}-\mathbf{k}_{2}=\left(\mathbf{q}_{1}-\mathbf{q}_{2}, \beta_{q_{1}}-\beta_{q_{2}}\right)$ between the two plane waves. In some materials $E_{p}$ also has a small angular dependence through $\epsilon_{r}{ }^{14}$

Since $r_{\text {eff }}$ is always real, the phase shift of $\hat{\delta n}\left(\mathbf{q}_{1}, \mathbf{q}_{2}\right)$ with respect to the interference grating $\mathbf{K}_{g}$ is dictated solely by $E_{m}$, which can be split into its real and imaginary parts, $E_{m}=-\left(E_{1}+i E_{2}\right)$, where

$$
\begin{aligned}
& E_{1}=\frac{E_{0}}{\left(E_{0} / E_{p}\right)^{2}+\left[\left(E_{p} / E_{d}\right)+1\right]^{2}}, \\
& E_{2}=\frac{E_{p}\left(E_{0}{ }^{2}+E_{d}{ }^{2}+E_{d} E_{p}\right)}{E_{0}{ }^{2}+\left(E_{d}+E_{p}\right)^{2}} .
\end{aligned}
$$

Note that in the planar (two-dimensional) cases, where $\boldsymbol{\beta}_{q_{1}}-\boldsymbol{\beta}_{q_{2}} \ll\left|q_{1}-q_{2}\right|, \mathbf{K}_{\mathrm{g}}$ is odd under both exchanges $\left(q_{1}, q_{2}\right) \rightarrow\left(q_{2}, q_{1}\right)$ and $\left(q_{1}, q_{2}\right) \rightarrow\left(-q_{1},-q_{2}\right)$, and $E_{2}$ behaves similarly, whereas $E_{1}$ is always even. These symmetry properties entail design considerations for a proper choice of the photorefractive crystalline medium and the direction of propagation, polarization, and application of external field, which are addressed below.

When more than two plane waves are present, $\delta n(\mathbf{r}, z)$ involves a summation over all possible interacting pairs of plane waves. For a given light beam $A(\mathbf{r}, z)$, which consists of a continuous spatial frequency spectrum of plane waves $f(\mathbf{q}, z)$, this summation takes the integral form

$$
\begin{aligned}
\delta n(\mathbf{r}, z)= & \frac{1}{|A(\mathbf{r}, z)|^{2}} \int \mathrm{d} \mathbf{q}_{1} \int \mathrm{d} \mathbf{q}_{2} f\left(\mathbf{q}_{1}, z\right) f^{*}\left(\mathbf{q}_{2}, z\right) E\left(\mathbf{q}_{1}, \mathbf{r}\right) \\
& \times E^{*}\left(\mathbf{q}_{2}, r\right) \exp \left[i\left(\beta_{q_{1}}-\beta_{q_{2}}\right) z\right] \delta \hat{\delta n}\left(\mathbf{q}_{1}, \mathbf{q}_{2}\right) .
\end{aligned}
$$

Note that, since the PR nonlinearity is independent of the 
absolute light intensity, $\delta n$ is normalized by the factor $|A(\mathbf{r}, z)|^{2}$. In addition, a constant factor that represents the dark irradiance ${ }^{15}$ may be added to the light intensity in the denominator of Eq. (8) in order to avoid unrealistic divergence of $\delta n$ in dark regions. Our expression for $\delta n$ is based on the assumption that the induced gratings are of low modulation depth and hence can be linearly superposed. This assumption, however, is not a major restriction when a beam is considered, provided that the beam has a small angular divergence. Our representation of the PR light-induced index change fails either in the proximity of dark spots or at the margins of the beam, where the local light intensity $|A(\mathbf{r}, z)|^{2}$ is of the order of the dark irradiance; there high-modulation-depth effects become significant, ${ }^{16}$ and the linearization assumption is no longer valid. We restrict our analysis to regions in which the light intensity is much higher than the dark irradiance, and thus we neglect the additional constant in the denominator of Eq. (8). This model of beam propagation in photorefractive media proved to be effective in the interpretation of a variety of wave-mixing processes ${ }^{13,14,17}$ and, in particular, was used for predicting a number of new phenomena (such as incoherent backscattering ${ }^{18}$ ).

\section{PHOTOREFRACTIVE SOLITON EQUATION}

We define a self-trapped optical beam (spatial soliton) as a wave form that does not change its shape during propagation along the optical axis $z$, that is,

$$
A(\mathbf{r}, z)=U(\mathbf{r}) \exp (i \gamma z),
$$

where $U(\mathbf{r})$, the transverse amplitude, and $\gamma$, the characteristic soliton propagation constant, may be complex (allowing for amplification or decay). Since diffraction is basically a symmetric process, the simplest way to compensate for it and to generate a spatial soliton in a PR medium is through a symmetric nonlinear process. Hence we require that $\delta n(\mathbf{r}, z)=\delta n(-\mathbf{r}, z)$ for all $\mathbf{r}$ and $z$. The substitution of this relation into Eq. (3) implies that

$$
U(\mathbf{r})= \pm U(-\mathbf{r}) .
$$

The antisymmetric solutions for $U(\mathbf{r})$ involve zero intensity at the origin and hence cannot be dealt with in the framework of our model (see the above limitations involving the dark irradiance). Therefore we discuss only symmetric solutions, which require that $\hat{\delta} n\left(\mathbf{q}_{1}, \mathbf{q}_{2}\right)=$ $\delta n\left(-\mathbf{q}_{1},-\mathbf{q}_{2}\right)$. Note that an antisymmetric nonlinearity $[\delta n(\mathbf{r}, z)=-\delta n(-\mathbf{r}, z)]$ does not satisfy Eq. (3) with a symmetric soliton waveform and hence does not provide any additional solutions.

In the most general case, $\hat{\delta}\left(\mathbf{q}_{1}, \mathbf{q}_{2}\right)$ can be expressed as

$$
\hat{\delta} n\left(\mathbf{q}_{1}, \mathbf{q}_{2}\right)=\iint g\left(\boldsymbol{\rho}, \boldsymbol{\rho}^{\prime}\right) \exp \left[-i\left(\mathbf{q}_{1} \cdot \boldsymbol{\rho}+\mathbf{q}_{2} \cdot \boldsymbol{\rho}^{\prime}\right) \mathrm{d} \boldsymbol{\rho} \mathrm{d} \boldsymbol{\rho}^{\prime},\right.
$$

so that, from Eqs. (1) and (8), we get

$$
\begin{aligned}
\delta n(\mathbf{r}, z)= & \frac{1}{|A(\mathbf{r}, z)|^{2}} \\
& \times \iint A(\mathbf{r}-\boldsymbol{\rho}, z) A^{*}\left(\mathbf{r}+\boldsymbol{\rho}^{\prime}, z\right) g\left(\boldsymbol{\rho}, \boldsymbol{\rho}^{\prime}\right) \mathrm{d} \boldsymbol{\rho} \mathrm{d} \boldsymbol{\rho}^{\prime} .
\end{aligned}
$$

Note the explicit nonlocal nature of the PR effect, which is brought out by the numerator in Eq. (12), and the local behavior of the damping factor $|A(\mathbf{r}, z)|^{2}$ in the denominator. The last argument was examined carefully and verified experimentally by Mamaev and Shkunov. ${ }^{15}$ When Eq. (12) is inserted into Eq. (3), the equation of evolution of the electromagnetic field reads as

$$
\begin{aligned}
\left(\frac{\partial}{\partial z}\right. & \left.-\frac{i}{2 k} \nabla_{\mathbf{r}}^{2}\right) A(\mathbf{r}, z) \\
& =\frac{i k}{n_{1}} \frac{1}{A^{*}(\mathbf{r}, z)} \iint A(\mathbf{r}-\boldsymbol{\rho}, z) A^{*}\left(\mathbf{r}+\boldsymbol{\rho}^{\prime}, z\right) g\left(\boldsymbol{\rho}, \boldsymbol{\rho}^{\prime}\right) \mathrm{d} \boldsymbol{\rho} \mathrm{d} \boldsymbol{\rho}^{\prime} .
\end{aligned}
$$

In particular, if we look for soliton solutions, we substitute Eq. (9) into Eq. (13) and get

$$
\begin{aligned}
(\gamma & \left.-\frac{1}{2 k} \nabla_{\mathbf{r}}^{2}\right) U(\mathbf{r}) \\
= & \frac{k}{n_{1}} \frac{1}{U^{*}(\mathbf{r})} \iint U(\mathbf{r}-\boldsymbol{\rho}) U^{*}\left(\mathbf{r}+\boldsymbol{\rho}^{\prime}\right) g\left(\boldsymbol{\rho}, \boldsymbol{\rho}^{\prime}\right) \mathrm{d} \boldsymbol{\rho} \mathrm{d} \boldsymbol{\rho}^{\prime} .
\end{aligned}
$$

The integrodifferential equation (14) can be transformed into an ordinary differential equation by a Taylor expansion of $U(\mathbf{r}-\boldsymbol{\rho})$ near $\boldsymbol{\rho}=0$ :

$$
U(\mathbf{r}-\boldsymbol{\rho})=U(\mathbf{r})-\nabla_{\mathbf{r}} U(\mathbf{r}) \cdot \boldsymbol{\rho}+1 / 2\left[\nabla_{\mathbf{r}} \nabla_{\mathbf{r}} U(\mathbf{r})\right]: \boldsymbol{\rho} \boldsymbol{\rho}+\ldots,
$$

along with an analogous expansion for $U^{*}\left(\mathbf{r}+\boldsymbol{\rho}^{\prime}\right)$ near $\boldsymbol{\rho}^{\prime}=0$, inserted into the right-hand side of Eq. (14).

The smallness parameter in the Taylor expansion [Eq. (15)] is given by $\epsilon=d / l$, where $d$ is the typical scale of nonlocality [dictated by the form of $\delta n\left(\mathbf{q}_{1}, \mathbf{q}_{2}\right)$ ] and $l$ is the characteristic transverse dimension of the beam. To truncate the expansion at a given order, one has to assume that $\epsilon<1$. Note that, because of the invariance of $\delta n(\mathbf{r}, z)$ under the exchange $\mathbf{r} \rightarrow-\mathbf{r}$, the odd-order terms of Eq. (15) do not contribute to the right-hand side of Eq. (14).

\section{TWO-DIMENSIONAL CASE}

Any photorefractive material is a noncentrosymmetric crystalline medium; such media do not have cylindrical symmetry; as a result the propagation equation is complicated in the general three-dimensional case. We reduce the problem to two dimensions, permitting diffraction in the $y$ direction and looking for self-trapping in only the $x$ direction or, alternatively, examining self-trapping in a slab PR waveguide, as shown in Fig. 1 . In this case $U(\mathbf{r})=U(x)$, and we obtain, by truncating the Taylor expansion after the second term,

$$
\begin{aligned}
\left(\frac{\partial}{\partial z}-\frac{i}{2 k} \frac{\partial^{2}}{\partial x^{2}}\right) A(x, z)= & \frac{-i k}{n_{1}} I_{11} \frac{1}{A^{*}}\left|\frac{\partial A}{\partial x}\right|^{2}+\frac{i k}{2 n_{1}} I_{20} \frac{\partial^{2} A}{\partial x^{2}} \\
& +\frac{i k}{2 n_{1}} I_{02} \frac{A}{A^{*}} \frac{\partial^{2} A^{*}}{\partial x^{2}} \\
& +\frac{i k}{4 n_{1}} I_{22} \frac{1}{A^{*}}\left|\frac{\partial^{2} A}{\partial x^{2}}\right|^{2}
\end{aligned}
$$

with 

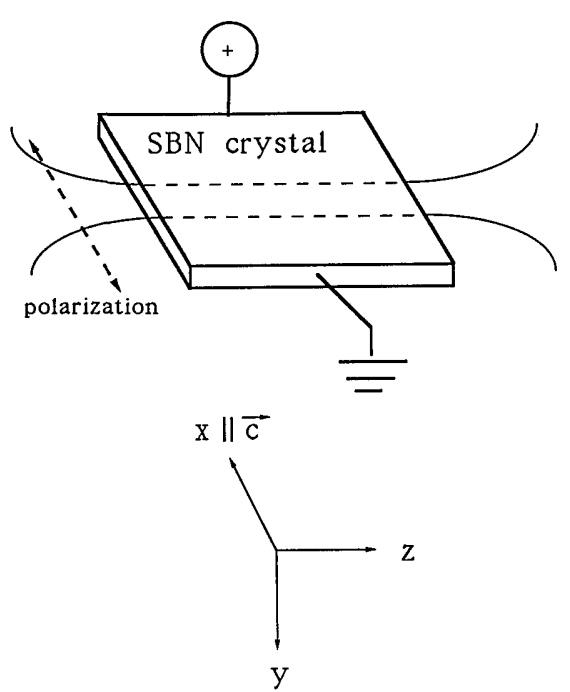

Fig. 1. Schematic experimental geometry for the observation of a PR spatial soliton in SBN.

$$
\begin{aligned}
I_{m n} & =\int \mathrm{d} \rho \int \mathrm{d} \rho^{\prime} g\left(\rho, \rho^{\prime}\right) \rho^{m} \rho^{\prime n} \\
& =\left.\exp [i(m+n) \pi / 2] \frac{\partial^{m}}{\partial q_{1}{ }^{m}} \frac{\partial^{n}}{\partial q_{2}{ }^{n}} \hat{\delta n}\left(q_{1}, q_{2}\right)\right|_{q_{1}=q_{2}=0}
\end{aligned}
$$

If we expand

$$
\hat{\delta n}\left(q_{1}, q_{2}\right)=\sum_{n=0}^{\infty} \sum_{m=0}^{\infty} a_{m n} q_{1}{ }^{m} q_{2}^{n},
$$

then

$$
I_{m n}=m ! n ! a_{m n} \exp [i(m+n) \pi / 2] .
$$

Under the symmetry condition $\hat{\delta} n\left(q_{1}, q_{2}\right)=\hat{\delta} n\left(-q_{1},-q_{2}\right)$, it follows that $a_{m n}=0$ if $m+n$ is odd. Besides, using the relation $\hat{\delta} n\left(q_{1}, q_{2}\right)=\hat{\delta} *\left(q_{2}, q_{1}\right)$ [from the reality of the perturbation in the refractive index $\delta n(x, z)]$, we obtain $a_{m n}=a_{n m} *$ and hence $I_{m n}=I_{n m} * \exp [i(m+n) \pi]$.

Let us discuss first the case in which the phase does not change in the transverse direction, i.e., $U(x)$ is real, while the propagation constant is allowed to be complex: $\gamma=\gamma_{1}+i \gamma_{2}$. By writing the real and the imaginary parts of Eq. (16) we get, after setting $I_{m n}=I_{m n}^{(\mathrm{re})}+i I_{m n}^{(\mathrm{im})}$,

$$
\begin{aligned}
\gamma_{1} U= & \frac{-k}{2 n_{1}} I_{20}^{(\mathrm{im})} \frac{\mathrm{d}^{2} U}{\mathrm{~d} x^{2}}, \\
\gamma_{2} U-\frac{1}{2 k} \frac{\mathrm{d}^{2} U}{\mathrm{~d} x^{2}}= & \frac{-k}{n_{1}} I_{11} \frac{(\mathrm{d} U / \mathrm{d} x)^{2}}{U} \\
& +\frac{k}{2 n_{1}}\left(I_{20}^{(\text {re) }}+I_{02}^{(\text {(re) })} \frac{\mathrm{d}^{2} U}{\mathrm{~d} x^{2}}\right. \\
& +\frac{k}{4 n_{1}} I_{22}\left(\frac{1}{U} \frac{\mathrm{d}^{2} U}{\mathrm{~d} x^{2}}\right)^{2}
\end{aligned}
$$

From the symmetry conditions of $I_{m n}$ 's, we get $I_{20}^{(\mathrm{im})}=$ $-I_{02}^{(\mathrm{im})}, I_{20}^{(\mathrm{re})}=I_{02}^{(\mathrm{re})}$, while $I_{m m}$ is real for all $m$. Thus Eq. (20) yields $\gamma_{1} U=0$, that is, $\gamma_{1}=0$ for every nonzero solution, while Eq. (21) becomes

$$
\begin{aligned}
\gamma_{2} U-\frac{1}{2 k} \frac{\mathrm{d}^{2} U}{\mathrm{~d} x^{2}}= & \frac{-k}{n_{1}} I_{11} \frac{(\mathrm{d} U / \mathrm{d} x)^{2}}{U} \\
& +\frac{k}{n_{1}} I_{20}^{(\mathrm{re})} \frac{\mathrm{d}^{2} U}{\mathrm{~d} x^{2}}+\frac{k}{4 n_{1}} I_{22}\left(\frac{1}{U} \frac{\mathrm{d}^{2} U}{\mathrm{~d} x^{2}}\right)^{2}
\end{aligned}
$$

Thus, whatever the character of $\hat{\delta}\left(q_{1}, q_{2}\right)$ (real or complex), the equation governing the evolution of $U$ is

$$
\gamma U-\frac{1}{2 k} U^{\prime \prime}=\frac{-k}{n_{1}} I_{11} \frac{U^{\prime 2}}{U}+\frac{k}{n_{1}} I_{20}^{(\mathrm{re})} U^{\prime \prime}+\frac{k}{4 n_{1}} I_{22} \frac{U^{\prime \prime 2}}{U},
$$

where $\gamma=\gamma_{2}$ (real) and $U^{\prime}$ and $U^{\prime \prime}$ indicate the first and the second derivatives of $U$ with respect to $x$.

At this point it is necessary to recall the expression of $\hat{\delta}\left(q_{1}, q_{2}\right)$ in the two-dimensional case [see Eqs. (5)(7)]. The interference grating vector obeys the relation $\mathbf{K}_{g}\left(q_{1}, q_{2}\right) \cong\left(q_{1}-q_{2}\right) \hat{x}$, so that $E_{2}\left(q_{1}, q_{2}\right)=-E_{2}\left(-q_{1}\right.$, $\left.-q_{2}\right)$, while $E_{1}\left(q_{1}, q_{2}\right)=E_{1}\left(-q_{1},-q_{2}\right)$. Since the coefficient $\hat{\delta} n\left(q_{1}, q_{2}\right)$ is proportional to the product $\left(E_{1}+\right.$ $\left.i E_{2}\right) * r_{\text {eff }}$ [see Eq. (5)], the symmetry requirement $\hat{\delta} n\left(q_{1}, q_{2}\right)=\hat{\delta} n\left(-q_{1},-q_{2}\right)$ can be satisfied either by the in-phase perturbation in the refractive index (generated by $E_{1}$ and responsible for the real parts of $I_{m n}$ ) or by the $\pi / 2$-phase-shifted gratings (generated by $E_{2}$ and responsible for the imaginary parts of $I_{m n}$ ) but not by both types of grating. For the solitary-wave solutions (where $U$ is real) it follows from Eq. (23) that the coefficients $I_{m n}$ (as high as the second order) must be real. We therefore require in the design considerations that $r_{\mathrm{eff}}\left(q_{1}, q_{2}\right)=$ $r_{\text {eff }}\left(-q_{1},-q_{2}\right)$, and it immediately follows that all the imaginary parts of $I_{m n}$ must be zero, since they do not satisfy this requirement.

When the first significant term is kept, Eq. (23) becomes

$$
\gamma-a \frac{U^{\prime \prime}}{U}+b\left(\frac{U^{\prime}}{U}\right)^{2}=0
$$

where

$$
\begin{aligned}
& a=\frac{1}{2 k}+\frac{k}{n_{1}} I_{20}^{(\mathrm{re})}, \\
& b=\frac{k}{n_{1}} I_{11} .
\end{aligned}
$$

To solve Eq. (24), we perform the change of dependent variable

$$
y=\frac{U^{\prime}}{U}
$$

which transforms it into

$$
a y^{\prime}+(a-b) y^{2}-\gamma=0
$$

or, equivalently,

$$
\frac{\mathrm{d} y}{A-y^{2}}=\frac{\mathrm{d} x}{D}
$$

where we set $A=\gamma /(a-b)$ and $D=a /(b-a)$.

A class of solutions of Eq. (29), subject to the symmetry condition $U^{\prime}(0) / U(0)=y(0)=0$, is given by

$$
y=\sqrt{A} \tanh (\alpha x),
$$


with

$$
\alpha=-\sqrt{A} / D,
$$

so that, after we recall Eq. (27),

$$
U(x)=U_{0}[\operatorname{sech}(\alpha x)]^{D} .
$$

If we require $U$ to go to zero when $x$ goes to infinity, then $D$ has to be positive; that is, after we set $a=\eta b / 2$,

$$
D=\frac{a}{b-a}=\frac{\eta}{2-\eta}>0,
$$

which in turn implies that $0<\eta<2$.

In particular, if we assume that $D=1$, that is, $a=b / 2$ or, equivalently, $\eta=1$, then we have the hyperbolic-secant solution

$$
U(x)=U_{0} \operatorname{sech}(\alpha x) .
$$

By recalling the expressions for $A$ and $D$, we can finally write

$$
\gamma=\frac{a^{2}}{a-b} \alpha^{2},
$$

so that Eq. (32) represents a general class of solutions of Eq. (24) for any $\alpha$ and $U_{0}$, provided that $D$ is given by relation (33), with the eigenvalue $\gamma$ determined by Eq. (35). In practice the condition $\epsilon \ll 1$ that is required for truncating the Taylor expansion of the field amplitude [see the paragraph after Eq. (15)] restricts the choice of $\alpha$ to values such that $\alpha d \ll 1$. More precisely, in order to put this intuitive criterion on firmer ground, we generalize Eq. (24) to include the term containing $U^{\prime \prime 2}$ [see Eq. (21)], thus getting

$$
\gamma^{\prime}+b\left(\frac{U^{\prime}}{U}\right)^{2}-a \frac{U^{\prime \prime}}{U}+c\left(\frac{U^{\prime \prime}}{U}\right)^{2}=0,
$$

where $c=\left(k / 4 n_{1}\right) I_{22}$. If we now assume, as suggested by Eq. (30), $U(x)$ to possess an asymptotic behavior of the kind $\exp (-\alpha D|x|)$, Eq. (36) furnishes

$$
\gamma^{\prime}=\gamma\left(1-\frac{c D^{2} \alpha^{2}}{a-b}\right)
$$

so that the smallest parameter of our expansion is given by $\epsilon^{\prime}=c D^{2} \alpha^{2} /(a-b)$.

Another class of solutions of Eq. (24) is given, as can be confirmed by direct substitution, by

$$
U(x)=U_{0} \exp \left(-\alpha^{2} x^{2}\right)
$$

for any $U_{0}$ and $\alpha$, provided that $a=b$, with the eigenvalue $\gamma$ being furnished in this case by the relation

$$
\gamma=-2 b \alpha^{2} .
$$

This solution cannot be obtained in the frame of the derivation that leads to the hyperbolic-secant-type pulse. In fact, for a Gaussian shape $y^{2}$ ranges from zero to infinity, so that Eq. (29) becomes singular for $y^{2}=A$. [Note that, according to the definition of $A$ and Eq. (35), $A>0$.]

Finally, we note that Eq. (24) does not admit of solutions of the dark solitary-wave kind, that is, solutions that tend asymptotically to a finite value for large values of $x$. In fact, since $U^{\prime} / U$ and $U^{\prime \prime} / U$ go to zero asymptotically while $U$ remains finite, the validity of Eq. (24) requires $\gamma$ to be zero. Under this circumstance Eq. (24) can be solved by quadrature, thus yielding the solution $y=1 /(1-a / b) x$, which obviously is not of the desired type.

The above considerations point to the existence of a lower bound on the material nonlinearity (corresponding to $\eta=0$ ). Below this lower bound diffraction overcomes self-focusing for all possible waveforms. In the solitarywave case there exists a lower bound on the product $E_{0} r_{\text {eff }}$ (the external field times the material Pockels coefficient) that is the so-called soliton threshold. It is derived from the requirement that $D>0$ (or, alternatively, that $\eta<2$ ), which is necessary to guarantee that the boundary condition at $|x| \rightarrow \infty$ is satisfied. More specifically, the soliton threshold is calculated from the condition

$$
\frac{1}{2 k}+\frac{k}{n_{1}} I_{20}^{(\text {re) }}<\frac{k}{n_{1}} I_{11}
$$

We elaborate this issue in the example in Section 5.

It is worth noting that the soliton threshold condition furnished by inequality (40) realistically expresses the well-known guidance condition ${ }^{19}$

$$
V=k r\left(2 n_{1} \Delta n\right)^{1 / 2} \geq 2
$$

for an optical waveguide of radius $r$. As an example, for a Gaussian PR soliton [see Eq. (38)] it is easy for one to show, after assuming $r \approx 1 / \alpha$, that relation (41) corresponds to

$$
\frac{1}{2 n_{1}{ }^{2} k}+\frac{k}{n_{1}} I_{20}^{\text {(re) }}<\frac{k}{n_{1}} I_{11},
$$

which is in practice equivalent to inequality (40).

It is well known ${ }^{11}$ that the efficiency of the PR effect is independent of the absolute light intensity. This property is expressed here through the normalization by $|A(\mathbf{r}, z)|^{2}$ in Eq. (8). The PR spatial solitons are therefore independent of their absolute intensity [ $U_{0}$ is arbitrary in Eqs. (27) and (28)]. The inclusion of material loss (gain) in our model results in a beam that maintains its transverse profile even as the total light intensity increases (gain) or attenuates (loss) with propagation. In the absorption case, for example, a linear term $\sigma A(\mathbf{r}, z)$ can be added to the left-hand side of Eq. (13), and the soliton propagation constant $\gamma$ can be allowed to be complex. If we take the imaginary part of $\gamma$ equal to $-\sigma$, we still get Eq. (14), with $\gamma$ replaced by its real part $\gamma_{r}$. The resulting solution is $U(\mathbf{r}) \exp \left(i \gamma_{r} z-\sigma z\right)$, and the transverse structure remains unchanged.

\section{DESIGN CONSIDERATIONS}

Several considerations have to be taken into account when one designs an experiment in order to observe PR spatial solitons. We describe here in detail the two-dimensional case of Section 4, while we consider the general threedimensional case in Section 6.

The first design consideration is the necessity for a real coupling coefficient $\hat{\delta}\left(q_{1}, q_{2}\right)$ for all $q_{1}$ and $q_{2}$. This can be achieved by applying an external dc field $E_{0}$ along the general direction of the grating vectors $K_{g}$, i.e., perpendicularly to the optical axis $z$. In this way we introduce a 
drift transport mechanism, which redistributes the optically excited charges, and the induced index grating is in phase with the interference grating for each pair of planewave components. The interacting pairs of plane waves are not capable of exchanging energy, but their phase is altered by the nonlinear coupling effects. Therefore the initial amplitudes of the waves remain constant, and their diffraction effects (which are due to accumulation of linear phases) can be compensated for by the nonlinear phase coupling. An alternative possibility is the use of the recently developed quadratic materials that belong to the potassium tantalate niobate group. ${ }^{20}$ These materials possess a surprisingly large $\mathrm{PR}$ nonlinearity with an externally applied electric field, which does not vanish even in the absence of this field, owing to a strain-induced Jahn-Teller effect. ${ }^{21}$ This zero-field PR effect results in the formation of a grating that is in phase with the intensity interference grating and hence does not support the energy-transfer process.

The second design consideration is the elimination of the imaginary part of $\hat{\operatorname{sn}}\left(q_{1}, q_{2}\right)$ for all $q_{1}$ and $q_{2}$, which in turn avoids the energy-transfer process and keeps the wave amplitudes fixed. In the conventional PR crystals this process is due to the unavoidable diffusion transport mechanism, which results in a $\pi / 2$ phase shift between the interference and the index gratings. Although this effect cannot be completely extinguished, its magnitude can be greatly reduced by a proper choice of the amplitude of the externally applied electric field $E_{0}$. Since the paraxial approximation is assumed in our model, the spatial spectrum $f(q, z)$ of the $\mathrm{PR}$ soliton is restricted to a relatively small angular deviation, and the resultant interference gratings (and the index gratings as well) have large periods. Consequently the limiting space-charge field $E_{p}$ is relatively large, and the diffusion field $E_{d}$ is small. Application of an appropriate external field, such that $\left|E_{d}\right| \ll\left|E_{0}\right| \ll\left|E_{p}\right|$, allows us to neglect the imaginary part of the coupling coefficient, so that

$$
E_{m}\left(q_{1}, q_{2}\right) \cong-E_{1}\left(q_{1}, q_{2}\right) \cong \frac{-E_{0}}{\left(E_{0} / E_{p}\right)^{2}+1}
$$

The alternative way of satisfying the above design requirement is to take advantage of the newly discovered effects in the paraelectric crystals ${ }^{20,21}$ in the absence of an externally applied electric field. Note that when the polarity of $E_{0}$ is reversed the linear diffraction and the nonlinear phase add up, and the diffraction effect is enhanced.

A third design consideration is associated with the symmetry requirement $\hat{\delta} n\left(q_{1}, q_{2}\right)=\hat{\delta} n\left(-q_{1},-q_{2}\right)$. As has been shown in Section 4, to satisfy this requirement for the real part of $\hat{\delta} n\left(q_{1}, q_{2}\right)$ one needs to choose the crystalline orientation and the beam polarization in such a way that $r_{\mathrm{eff}}\left(q_{1}, q_{2}\right)=r_{\mathrm{eff}}\left(-q_{1},-q_{2}\right)$. Since we are interested in a planar geometry, care must be taken to avoid anisotropic scattering ${ }^{8}$ in the crystal.

Another difficulty that may arise in experimental realization of the $\mathrm{PR}$ solitons is interaction with the fanning noise, which can alter the beam transverse structure and degrade the soliton. Fanning is present in all the efficient PR crystals that support the energy-coupling process [and hence have a nonzero imaginary part of $\hat{\delta n}\left(q_{1}, q_{2}\right)$. Scattered light is amplified because of this process at the expense of the input beam. ${ }^{13}$ Eliminating the imaginary part of the coupling coefficient prevents the evolution of the fanning and avoids the problem. Should residual $\pi / 2$-phase-shifted gratings exist [Eq. (43)], the choice of a transversely narrow soliton waveform reduces the cross section of interaction with the noise, and the fanning all but disappears. [In Ref. 13 it was demonstrated that a choice of a small-spot-size Gaussian beam input suppresses the fanning completely, even in the presence of a large imaginary part of $\hat{\delta n}\left(q_{1}, q_{2}\right)$.]

\section{PROPERTIES OF THE PHOTOREFRACTIVE SPATIAL SOLITON}

Since the nonlinearity responsible for the self-trapping effects is associated with the PR effect, the properties of the PR spatial soliton significantly differ from those of the Kerr-like soliton, with the only common feature being the diffraction-free propagation in the nonlinear medium.

The major distinguishing feature of the PR spatial soliton is that the propagating waveform is unchanged in the medium, at high or low light intensities and at all levels between; the waveform has the same shape, regardless of the absolute light intensity. This property, which is due to the independence of the efficiency of the PR effect with regard to the absolute light intensity, is expressed by the presence of the term $|A(\mathbf{r}, z)|^{2}$ in the denominator on the right-hand side of Eq. (8). For the same reason the PR solitons can accommodate material loss (absorption) or gain (amplification) as described at the end of Section 4.

Another property originates from the fact that the PR gratings that are required for the existence of the solitarywave solutions are in phase with their corresponding interference gratings and hence do not support changes in the amplitudes of the plane-wave components. In the absence of the energy-exchange process, the PR spatial solitons preserve their original shape and their uniform phase. For the same reason an arbitrary input profile cannot evolve into the soliton shape, as it could with temporal Kerr solitons. In order to obtain a PR soliton, one has to start with the correct waveform. A degree of fine tuning of the PR effect [and hence of the soliton shape, i.e., of the value of $D$ in Eq. (32)] is made possible by varying the externally applied electric field $E_{0}$. The stability of the PR soliton with regard to small deviations from the proper solution and to small perturbations in the refractive index $n_{1}$ is currently under study.

\section{EXAMPLE: SPATIAL SOLITON IN STRONTIUM BARIUM NIOBATE}

As an example, we describe the design of a photorefractive spatial soliton in SBN. The symmetry requirement $r_{\text {eff }}\left(q_{1}, q_{2}\right)=r_{\text {eff }}\left(-q_{1},-q_{2}\right)$ is satisfied by the choice of an optical field that is linearly polarized along $x$, once the $x$ direction is adjusted to coincide with the crystalline c axis (we neglect the relatively small Pockels coefficients, other than $r_{33}$ ), which yields

$$
\begin{aligned}
\hat{\delta n}\left(q_{1}, q_{2}\right) & \cong \frac{n_{1}{ }^{3}}{2} r_{33} E_{0} \frac{1}{1+\left(\frac{E_{0} \epsilon_{0} \epsilon_{r}}{e P_{d}}\right)^{2}\left(q_{1}-q_{2}\right)^{2}} \\
& \equiv \frac{B}{1+d^{2}\left(q_{1}-q_{2}\right)^{2}} .
\end{aligned}
$$




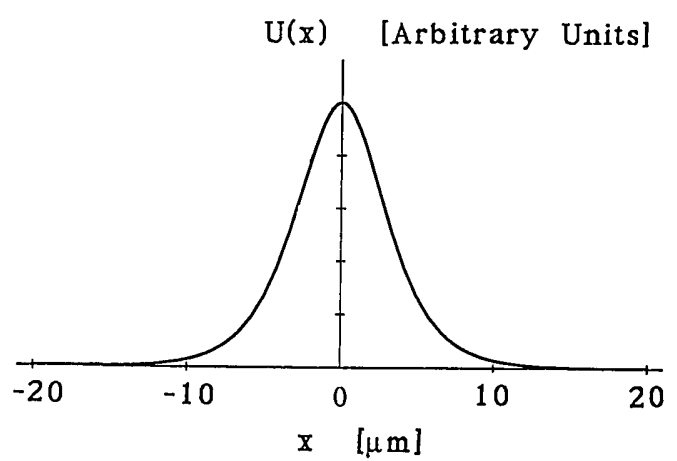

Fig. 2. Plot of the cross section of the light field amplitude $U(x)$ of the PR soliton given as an example in the text. The vertical axis gives the amplitude in arbitrary units, and the horizontal axis $x$ is in micrometers.

The parameters $e, P_{d}, \epsilon$, and $\epsilon_{r}$ were defined in Section 2. The extension region of influence of the nonlocal PR effect, as discussed at the end of Section 3 , is associated with the parameter $d$ that appears in relation (44).

For comparison, note that, if one works out the $r_{\text {eff }}$ coefficient for propagation along the crystalline c axis $(z \| \mathbf{c})$ and polarization in the $x$ direction, one has

$$
r_{\mathrm{eff}}\left(q_{1}, q_{2}\right)=r_{33} \frac{q_{1} q_{2}\left(q_{1}+q_{2}\right)}{2 k^{3}},
$$

which results in $\operatorname{Im}\left[\hat{\delta n}\left(q_{1}, q_{2}\right)\right]=\operatorname{Im}\left[\hat{\delta n}\left(-q_{1},-q_{2}\right)\right]$; however, the real part of $\hat{\delta}\left(q_{1}, q_{2}\right)$ is odd under the exchange $\left(q_{1}, q_{2}\right) \rightarrow\left(-q_{1},-q_{2}\right)$ and hence does not satisfy the design requirements of the $\mathrm{PR}$ spatial soliton. This geometry is used primarily for nonlinear mode-coupling effects in $\mathrm{PR}$ waveguides, ${ }^{14,22,23}$ where efficient symmetrical energy coupling from high-order waveguide modes to lowerorder modes takes place. \{For a detailed discussion of the symmetry of $\operatorname{Im}\left[\hat{\delta} n\left(q_{1}, q_{2}\right)\right]$ in this case, see Ref. 22.$\}$

The coefficients $I_{11}$ and $I_{20}^{(\text {re) }}$ can be found, according to their definitions in Eqs. (25) and (26), to be $I_{11}=$ $-I_{20}^{(\mathrm{re})}=-2 B d^{2}$. The third-order term in the Taylor expansion of Eqs. (15)-(17) is identically zero, and the fourth-order term corresponds to the coefficient $I_{22}=$ $-I_{40}^{(\mathrm{re})}=-I_{04}^{(\mathrm{re})}=2 B d^{4}$.

The soliton threshold is manifested by a positive value of $D$ in Eq. (32). This condition implies a negative value of $B$ (and thus of the externally applied electric field $E_{0}$ ). The soliton threshold corresponds to

$$
\frac{-n_{1}}{4 k^{2} d^{2}}<B<\frac{-n_{1}}{8 k^{2} d^{2}}
$$

and therefore $1 /\left(4 n_{1}{ }^{2} k^{2} d^{2}\right)<r_{33}\left|E_{0}\right|<1 /\left(2 n_{1}{ }^{2} k^{2} d^{2}\right)$. Recalling that $d=E_{0} \epsilon_{r} \epsilon_{0} / e P_{d}$ and $k=2 \pi n_{1} / \lambda$ yields the condition

$$
\left(\frac{\lambda^{2} e^{2} P_{d}^{2}}{16 \pi^{2} r_{33} n_{1}{ }^{4} \epsilon_{0}{ }^{2} \epsilon_{r}{ }^{2}}\right)^{1 / 3}<\left|E_{0}\right|<\left(\frac{\lambda^{2} e^{2} P_{d}^{2}}{8 \pi^{2} r_{33} n_{1}{ }^{4} \epsilon_{0}{ }^{2} \epsilon_{r}{ }^{2}}\right)^{1 / 3} \text {. }
$$

When we take a value of $224 \times 10^{-12} \mathrm{~m} / \mathrm{V}$ for $r_{33}, \lambda \approx$ $0.5 \mu \mathrm{m}$, and the parameters $\epsilon_{r} \approx 1100, n_{1}=2.35, P_{d}=4 \times$ $10^{16} \mathrm{~cm}^{-3}$ yields that the magnitude of the electric field that can induce self-trapping of a light beam in the above SBN crystal is between the values 4.6 and $5.85 \mathrm{kV} / \mathrm{cm}$.
As an example, we have calculated the factor $d$ for $\left|E_{0}\right|=5 \mathrm{kV} / \mathrm{cm}$ and for the above parameters $\epsilon_{r}, n_{1}$, and $P_{d}$, thus getting $d \sim 0.76 \mu \mathrm{m}, B \approx-7.27 \times 10^{-4}$, and therefore $\eta \approx 1.21$ and $D \approx 1.53$. The neglect of the fourth-order term, along with higher-order terms, in Eqs. (15) and (16) is justified when the condition $\alpha d \ll 1$ is satisfied, which can be done by choosing $\alpha$ to be a small fraction of $k$.

A schematic experimental geometry for the observation of a PR spatial soliton in SBN is shown in Fig. 1. The soliton waveform that is pertinent to this configuration is $U(x)=U_{0} \operatorname{sech}^{D}(\alpha x)$ for the (above) value of $D \approx 1.53$ and the choice of $\alpha=0.3 \mu \mathrm{m}^{-1}$; this waveform is shown in Fig. 2.

\section{DISCUSSION}

In this paper we have studied the possibility for a light beam to be self-trapped in a photorefractive medium. We have developed the formalism for the most general case and have provided a detailed study of the simple, twodimensional spatial soliton with a uniform transverse phase (solitary wave). The full spectrum of solutions and their properties and existence conditions were given together with the pertinent design requirements.

There remains, however, a wide range of open questions and various directions for further extensive research. Some questions arise from analytical problems connected with soliton solutions with transverse nonuniform phases, incorporation of nonuniform losses, stability, collisions between solitons, dark solitons, and so on. Other questions, such as the existence of a three-dimensional soliton, are generated by the physical complexity of our problem. Since the nonlinear medium in our case is always a crystal that does not have cylindrical symmetry, the extension of our model to three dimensions is not an easy task. The last, and probably the most interesting, direction of future research is the experimental challenge to realize a photorefractive spatial soliton. As calculated in our example, the required external field is within the current experimental feasibility.

\section{ACKNOWLEDGMENTS}

This research was supported by the U.S. Army Office of Scientific Research and the by U.S. Air Force Office of Scientific Research.

\section{REFERENCES}

1. M. Hercher, J. Opt. Soc. Am. 54, 563 (1964).

2. R. Y. Chiao, E. Garmire, and C. H. Townes, Phys. Rev. Lett. 13, 479 (1964).

3. V. E. Zakharov and A. B. Shabat, Sov. Phys. JETP 34, 62 (1972).

4. J. E. Bjorkholm and A. Ashkin, Phys. Rev. Lett. 32, 129 (1974).

5. A. Barthelemy, S. Maneuf, and C. Froehly, Opt. Commun. 55, 201 (1985).

6. J. S. Aitchinson, A. M. Weiner, Y. Silberberg, M. K. Oliver, J. L. Jackel, D. E. Leaird, E. M. Vogel, and P. W. Smith, Opt. Lett. 15, 471 (1990).

7. M. Segev, B. Crosignani, A. Yariv, and B. Fischer, Phys. Rev. Lett. 68, 923 (1992).

8. D. A. Temple and C. Warde, J. Opt. Soc. Am. B 3, 337 (1986). 
9. S. Solimeno, B. Crosignani, and P. Di Porto, Guiding, Diffraction and Confinement of Optical Radiation (Academic, Orlando, Fla., 1986), Chap. 8.

10. B. Crosignani and A. Yariv, J. Opt. Soc. Am. A 1, 1034 (1984).

11. J. O. White, S. K. Kwong, M. Cronin-Golomb, B. Fischer, and A. Yariv, in Photorefractive Materials and their Applications II, P. Günter and J.-P. Huignard, eds. (Springer-Verlag, Berlin, 1989), Chap. 4.

12. J. Feinberg and R. W. Hellwarth, Opt. Lett. 5, 519 (1980); 6, 257 (1981).

13. M. Segev, Y. Ophir, and B. Fischer, Opt. Commun. 77, 265 (1990).

14. M. Segev, Y. Ophir, and B. Fischer, Appl. Phys. Lett. 56, 1086 (1990).
15. A. V. Mamaev and V. V. Shkunov, Sov. J. Quantum Electron. 19, 1199 (1990).

16. F. Vachss and L. Hesselink, J. Opt. Soc. Am. A 5, 690 (1988).

17. G. C. Valley, J. Opt. Soc. Am. B 9, 1440 (1992).

18. M. Segev and A. Yariv, Opt. Lett. 16, 1938 (1991).

19. D. Marcuse, Theory of Dielectric Optical Waveguides (Academic, New York, 1974).

20. A. Agranat, V. Leyva, and A. Yariv, Opt. Lett. 14, 1017 (1989).

21. R. Hofmeister, A. Yariv, S. Yagi, and A. Agranat, Phys. Rev. Lett. 69, 1459 (1992).

22. B. Fischer and M. Segev, Appl. Phys. Lett. 54, 684 (1989).

23. F. Ito and K. Kitayama, Appl. Phys. Lett. 59, 1932 (1991). 